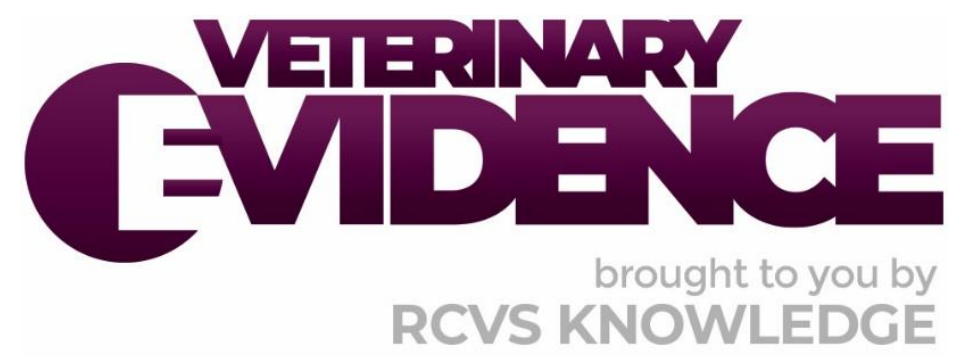

\title{
Tibial Diaphyseal Fracture Rates Following Tibial Tuberosity Advancement Rapid (TTAR) and Traditional Tibial Tuberosity Advancement (tTTA)
}

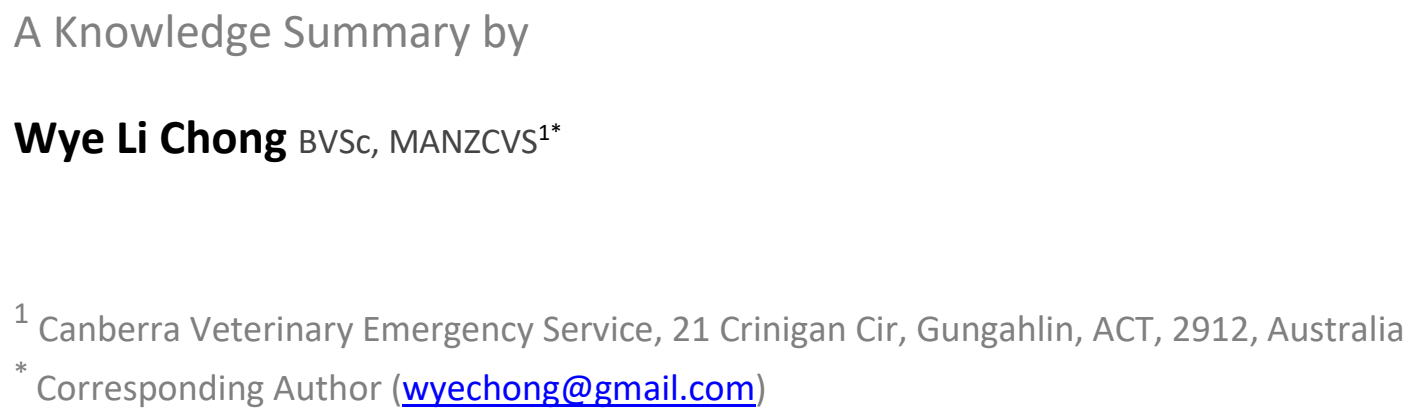

Wye Li Chong BVSc, MANZCVS ${ }^{1^{*}}$

${ }^{1}$ Canberra Veterinary Emergency Service, 21 Crinigan Cir, Gungahlin, ACT, 2912, Australia

*Corresponding Author (wyechong@gmail.com)

ISSN: 2396-9776

Published: 05 Mar 2019

in: Vol 4, Issue 1

DOI: http://dx.doi.org/10.18849/ve.v4i1.179

Reviewed by: Geoff Robins (BVetMed, FACVSc) and Yves

Samoy (DVM, PhD, DipECVSMR)

Next Review Date: 05 Mar 2020 


\section{KNOWLEDGE SUMMARY}

\section{PICO question}

In dogs with cruciate disease, is the use of TTA Rapid (TTAR) compared to traditional TTA (TTTA) associated with a higher risk of tibial diaphyseal fractures?

\section{Clinical bottom line}

Based on studies published between January 2013 and January 2018, the rate of tibial diaphyseal fractures as a complication of Tibial Tuberosity Advancement Rapid (TTAR) surgery is within the published limits of traditional Tibial Tuberosity Advancement (tTTA). In this period, seven studies were related to tTTA, comprising of four; one case series, two retrospective case-control studies, and one retrospective cohort study. Five case series were related to TTAR. All evidence within this period has been observational (Level 3 and 4 evidence). No direct head-to-head comparison between the techniques has been studied.

\section{The evidence}

Based on a literature search of the last 5 years (prior to 23 January 2018), there were seven PICO related studies for tTTA and five for TTAR. Of the articles for tTTA, 3/7 were retrospective case series, $1 / 7$ prospective case series, 2/7 retrospective case-controls and 1/7 retrospective cohort study. All TTAR studies were case series.

\begin{tabular}{|l|l|}
\hline Technique & Evidence \\
\hline tTTA & $\begin{array}{l}\text { Level } 4-\text { four studies } \\
\text { Level } 3 \text { - three studies }\end{array}$ \\
\hline TTAR & Level 4 - five studies \\
\hline
\end{tabular}

Table 1: A summary of the strength of evidence.

\section{Summary of the evidence}

\begin{tabular}{|c|c|c|}
\hline \multicolumn{3}{|l|}{ Costa et al., (2017) tTTA } \\
\hline Population: & Dogs & \\
\hline Sample size: & 1613 & \\
\hline Intervention details: & & $\begin{array}{l}\text { - All dogs received perioperative Cephazolin } 22 \mathrm{mg} / \mathrm{kg} \\
\text { - All dogs received traditional TTA procedure (KYON or } \\
\text { Medicatech Vet). }\end{array}$ \\
\hline
\end{tabular}

\section{Veterinary Evidence}




\begin{tabular}{|c|c|}
\hline & $\begin{array}{l}\text { - Meniscal release or meniscectomy was performed based } \\
\text { on preference. } \\
\text { - No bone grafts were placed on osteotomy site. } \\
\text { - All patients received postoperative coaptation. }\end{array}$ \\
\hline Study design: & Retrospective case-control study \\
\hline Outcome studied: & $\begin{array}{l}\text { Major complication rates in dogs receiving tibial tuberosity } \\
\text { advancement surgery and the effects of medial meniscal release on } \\
\text { postoperative meniscal tear. }\end{array}$ \\
\hline $\begin{array}{l}\text { Main findings: } \\
\text { (relevant to PICO question): }\end{array}$ & $\begin{array}{l}\text { Major complication rate }-\mathbf{1 3 . 4 \%} \\
\text { Minor complications - not included } \\
\text { Medial meniscal tear } \\
40.5 \% \text { had medial meniscal injury and were treated with partial } \\
\text { meniscectomy } \\
59.4 \% \text { had normal meniscus. } 72.4 \% \text { of these dogs had meniscal } \\
\text { release. } 0.5 \% \text { of these dogs developed postliminary meniscal injury. } \\
27.6 \% \text { of dogs with normal meniscus did not receive meniscal } \\
\text { release. } 10.2 \% \text { developed postliminary meniscal injury. } \\
\text { Postoperative patellar luxation } \\
1.2 \% \text { developed patellar luxation postoperatively. } \\
\text { Surgical site infection } \\
6.9 \% \text { of dogs developed superficial surgical site infection All were } \\
\text { managed successfully with conservative treatment. } 1.1 \% \text { developed } \\
\text { deep SSI necessitating surgical intervention. } \\
\text { Implant failure and fractures } \\
1 \% \text { of dogs had implant failure requiring revision surgery. } \\
0.7 \% \text { ( } 11 \text { dogs) developed fracture; } 3 / 11 \text { tibial diaphyseal fractures } \\
\text { and } 8 / 11 \text { tibial tuberosity fracture. } \\
\text { PICO - three (0.18\%) tibial diaphyseal fractures were reported. }\end{array}$ \\
\hline Limitations: & $\begin{array}{l}\text { - This study is retrospective and may be affected by } \\
\text { bias in case selection and suitability for TTA. } \\
\text { - Factors that could have affected surgical outcome } \\
\text { were not assessed, such as limb conformation or } \\
\text { body condition score. } \\
\text { - Minor complications were not assessed. } \\
\text { - Being retrospective in nature, this study confuses } \\
\text { the term incidence with prevalence. } \\
\text { - Did not correlate the use of perioperative antibiotics } \\
\text { and the development of surgical site infection. } \\
\text { - There was no power calculation. }\end{array}$ \\
\hline
\end{tabular}

\begin{tabular}{|r|l|}
\hline \multicolumn{2}{|l|}{ Danielson et al., (2016) tTTA } \\
\hline Population: & $\begin{array}{l}\text { Dogs receiving single-session bilateral plated tibial tuberosity } \\
\text { advancement. }\end{array}$ \\
\hline Sample size: & 148 stifle joints \\
\hline Intervention details: & Single stage bilateral traditional TTA. \\
\hline
\end{tabular}




\begin{tabular}{|c|c|}
\hline & $\begin{array}{l}68 \text { tTTAs were performed with forked plates. } \\
75 \text { tTTAs were performed with screw plates. }\end{array}$ \\
\hline Study design: & Retrospective case series \\
\hline Outcome studied: & $\begin{array}{l}\text { Short-term radiographic complication rates and healing scores in } \\
\text { bilateral single-session TTAs. }\end{array}$ \\
\hline $\begin{array}{l}\text { Main findings: } \\
\text { (relevant to PICO question): }\end{array}$ & $\begin{array}{l}\text { Overall radiographic complication rates of } 17.6 \% \text { ( } 13 / 72 \text { dogs), of } \\
\text { which only one dog was considered to have major complications. } \\
\text { Minor complications in } 12 \text { dogs include forked plate displacement } \\
\text { (6), tibial tuberosity fracture (4), screw failure (2) and cage } \\
\text { displacement (1). } \\
\text { Forked plates had greater complications compared to screw plates. } \\
\text { Four stifle joints had tibial tuberosity fractures. } \\
\text { PICO - One stifle joint had tibial diaphyseal fractures (0.007\%). }\end{array}$ \\
\hline Limitations: & $\begin{array}{l}\text { - The radiographic complications were evaluated by only one } \\
\text { radiologist. } \\
\text { - The radiographic scoring system is subjective and may be } \\
\text { prone to inter-operator variability. } \\
\text { - There is no control population (unilateral TTA) used for } \\
\text { comparison and testing of the null hypothesis. } \\
\text { - Comparison was based on results of other studies. } \\
\text { - The outcomes of patients with major and minor } \\
\text { complications were not reported. } \\
\text { - Non-radiographic complications were not reported. } \\
\text { - Statistical significance (P value) for comparing complications } \\
\text { between forked and screw plates was not reported. }\end{array}$ \\
\hline
\end{tabular}

\begin{tabular}{|c|c|}
\hline \\
\hline \multicolumn{2}{|c|}{$\begin{array}{l}\text { Proot \& Corr (2013) tTTA } \\
\qquad \text { Population: } \\
122 \text { dogs }\end{array}$} \\
\hline Sample size: & 167 stifle joints \\
\hline Intervention details: & $\begin{array}{l}\text { - All dogs received plated TTA by the same surgeon. } \\
\text { Dogs with meniscal pathology were partially or } \\
\text { completely resected. Dogs with intact meniscus were } \\
\text { left in situ. } \\
\text { - Consecutive records were plotted retrospectively using } \\
\text { cumulation summation technique (CUSUM) to measure } \\
\text { cumulative success for clinical audit and competence of } \\
\text { a single surgeon over time. }\end{array}$ \\
\hline Study design: & Retrospective case series \\
\hline Outcome studied: & $\begin{array}{l}\text { - Cumulative success rates of a single general practitioner } \\
\text { surgeon. } \\
\text { - Major and minor complication rates. }\end{array}$ \\
\hline
\end{tabular}




\begin{tabular}{|c|c|}
\hline $\begin{array}{l}\text { Main findings: } \\
\text { (relevant to PICO question): }\end{array}$ & 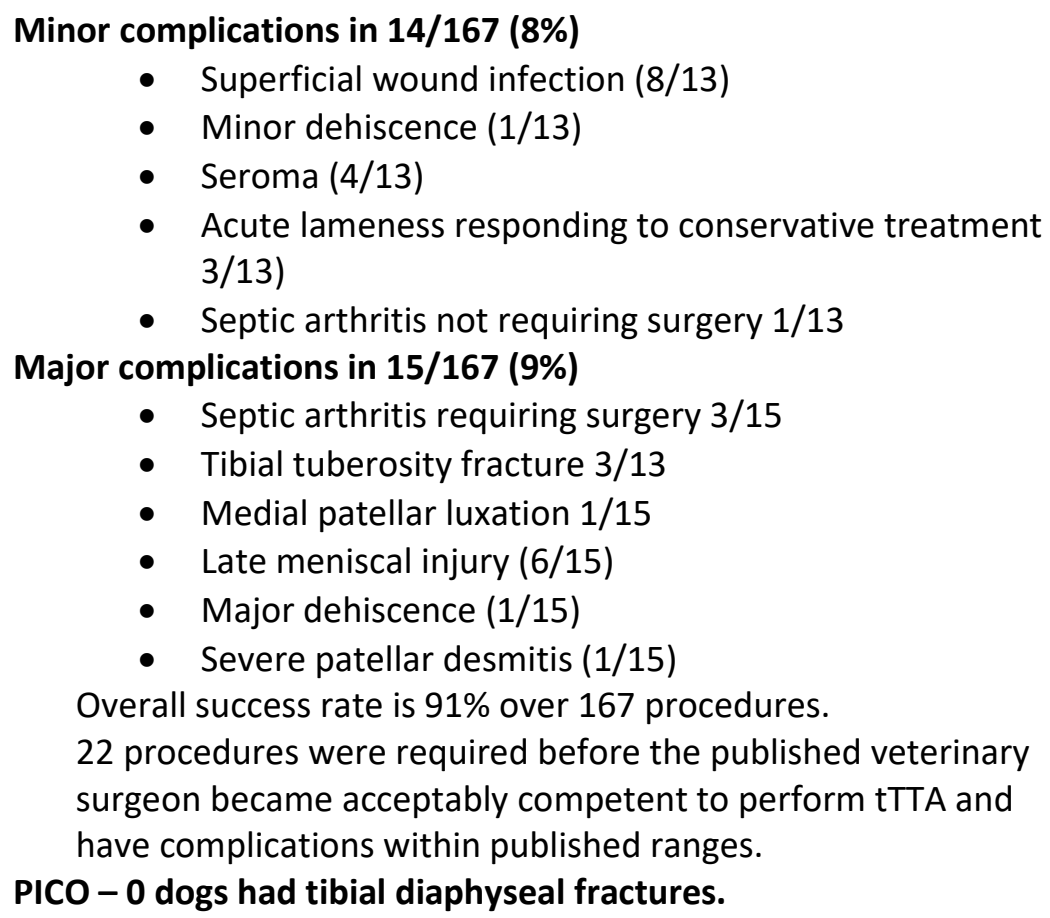 \\
\hline Limitations: & $\begin{array}{l}\text { - This paper only measured success as the absence of } \\
\text { complication rates, and not the clinical outcome. }\end{array}$ \\
\hline
\end{tabular}

\begin{tabular}{|c|c|}
\hline \multicolumn{2}{|l|}{ de Lima Dantas et al., (2016) tTTA } \\
\hline Population: & Boxer vs. non-Boxer dogs with cruciate disease \\
\hline Sample size: & 36 Boxer stifle joints and 271 non-Boxer stifle joints \\
\hline Intervention details: & $\begin{array}{l}\text { All dogs received forked plate TTA } \\
\text { Meniscus: } \\
\text { - } \quad 12 / 36 \text { Boxers and } 94 / 271 \text { non-Boxers had cranial cruciate } \\
\text { ligament remnant debrided. } \\
\text { - } \quad 8 / 36 \text { Boxers and } 91 / 271 \text { non-Boxers had medial meniscal } \\
\text { injury treated with partial meniscectomy. } \\
\text { - } 2 / 36 \text { Boxers and 15/271 non-Boxers had complete medial } \\
\text { meniscectomy. } \\
\text { - } \quad 1 / 36 \text { Boxers and } 3 / 271 \text { non-Boxers had medial meniscal } \\
\text { release. } \\
\text { - } 15 / 34 \text { Boxer and } 111 / 250 \text { non-Boxer stifle joints received } \\
\text { bone graft. } \\
\text { Antibiotics: } \\
\text { - } 11 / 30 \text { Boxers and } 123 / 264 \text { non-Boxers received amoxicillin- } \\
\text { clavulanic acid perioperatively. } \\
\text { - } 19 / 30 \text { Boxers and 141/264 non-Boxers received Cefuroxime } \\
\text { perioperatively. } \\
\text { 24/36 Boxers and 152/271 non-Boxers received antibiotics } \\
\text { postoperatively. }\end{array}$ \\
\hline
\end{tabular}




\begin{tabular}{|c|c|}
\hline Study design: & Retrospective cohort study \\
\hline Outcome studied: & $\begin{array}{l}\text { Incidence of major and minor complication rates associated with } \\
\text { tTTA in Boxers vs. non-Boxers. }\end{array}$ \\
\hline $\begin{array}{l}\text { Main findings: } \\
\text { (relevant to PICO question): }\end{array}$ & $\begin{array}{l}\text { - } 14 / 36 \text { ( } 38.9 \%) \text { Boxers and } 37 / 271(13.6 \%) \text { non-Boxers had } \\
\text { major complications }(P<0.001) \text {. } \\
\text { - } 6 / 36(16.7 \%) \text { Boxers and } 6 / 271(2.21 \%) \text { non-Boxers had } \\
\text { minor complications ( } P=0.003) \text {. } \\
\text { - } 4 / 36(11.1 \%) \text { Boxers and } 22 / 271(8.1 \%) \text { non-Boxers had late } \\
\text { meniscal injury }(P=0.52) \text {. } \\
\text { - } 7 / 36(19.4 \%) \text { Boxers and } 11 / 271 \text { (4.1\%) non-Boxers had } \\
\text { postoperative infections. } \\
\text { - } \text { Boxers compared to non-Boxers had an odds ratio of } 5.78 \text { of } \\
\text { developing complication. } \\
\text { - } 2 / 36(5.6 \%) \text { Boxers had tibial tuberosity fractures compared } \\
\text { to } 1 / 271(0.4 \%) \text { non-Boxers }(P=0.03) \text {. } \\
\text { PICO - } 0 \text { cases had tibial diaphyseal fractures. }\end{array}$ \\
\hline Limitations: & $\begin{array}{l}\text { - } \quad \text { Retrospective design predisposes the study population } \\
\text { - } \quad \text { Complication rates were determined based on medical } \\
\text { records of a referral hospital - this may have been } \\
\text { under- or over-estimated as it does not take into } \\
\text { consideration complication rates detected by a referring } \\
\text { veterinarian. } \\
\text { - This study includes complications by residents as well as } \\
\text { specialists, which may influence the incidence due to the } \\
\text { learning curve of residents. } \\
\text { - Small number of Boxer dogs compared to control } \\
\text { - } \text { population ( } 36 \text { vs. } 271 \text { ). } \\
\text { No randomisation or blinding, which can lead to bias. }\end{array}$ \\
\hline
\end{tabular}

\section{Kiefer et al., (2015) tTTA}

\begin{tabular}{|c|c|}
\hline Population: & $\begin{array}{l}\text { Dogs that underwent single-stage bilateral plated TTA vs. unilateral } \\
\text { plated TTA }\end{array}$ \\
\hline Sample size: & 44 (88) bilateral TTA (bTTA) vs. 86 unilateral TTA (UTTA) \\
\hline Intervention details: & $\begin{array}{l}\text { All dogs received plated TTA system - manufacturer undefined. } \\
\text { All dogs received commercial synthetic or cancellous bone graft } \\
\text { on osteotomy site. } \\
\text { Some dogs received external coaptation (number undefined). } \\
\text { All dogs were re-evaluated at: } \\
\text { - } 10 \text { weeks postoperatively to evaluate skin sutures } \\
\text { - } 4 \text { and } 8 \text { weeks for radiographs. }\end{array}$ \\
\hline Study design: & Retrospective case-control \\
\hline Outcome studied: & $\begin{array}{l}\text { Major and minor complication rates of bTTA compared to UTTA at } 4 \\
\text { and } 8 \text { weeks postoperatively. }\end{array}$ \\
\hline
\end{tabular}




\begin{tabular}{|c|c|}
\hline & $\begin{array}{l}\text { Major complications defined as complications requiring further } \\
\text { surgery. } \\
\text { Minor complications defined as any other complications. }\end{array}$ \\
\hline $\begin{array}{l}\text { Main findings: } \\
\text { (relevant to PICO question): }\end{array}$ & $\begin{array}{l}\text { Major complication rates (no significant difference) } \\
\text { bTTA } 11 / 88(12.5 \%) \text { vs. UTTA } 2 / 86 \text { ( } 2.3 \%) \\
\text { Minor complication rates (significantly different) } \\
\text { bTTA } 23 / 88 \text { ( } 26.1 \%) \text { vs. UTTA } 21 / 86(24.4 \%) \\
\text { Overall complication rate (no significant difference) } \\
\text { bTTA } 31 / 88(35.2 \%) \text { vs. UTTA } 21 / 86(24.4 \%) \\
\text { Fracture } \\
\text { bTTA - } 2 / 88 \text { tibial diaphyseal fracture and } 9 / 99 \text { tibial tuberosity } \\
\text { fracture } \\
\text { uTTA - } 1 / 88 \text { tibial tuberosity fracture } \\
\text { PICO - } 2 / 88 \text { (2.3\%) tibial diaphyseal fracture in bTTA and } \mathbf{0 / 8 8} \text { in } \\
\text { uTTA }\end{array}$ \\
\hline Limitations: & $\begin{array}{l}\text { - Retrospective design. } \\
\text { - tTTA procedure was modified over time so there could } \\
\text { have been more complications in earlier cases. } \\
\text { - Postoperative care was not standardised. } \\
\text { - } \text { Did not compare staged bTTA vs single-stage bTTA. } \\
\text { Relative risks were not reported. }\end{array}$ \\
\hline
\end{tabular}

\begin{tabular}{|c|c|}
\hline \\
\hline \multicolumn{2}{|c|}{$\begin{array}{l}\text { McDonald et al., (2013) tTTA } \\
\text { Population: }\end{array}$} \\
\hline Sample size: & 24 dogs \\
\hline Intervention details: & $\begin{array}{l}\text { - All dogs underwent arthrotomy or arthroscopy. } \\
\text { - No dogs received meniscal release. } \\
\text { - Partial meniscectomy performed in cases of meniscal injury. } \\
\text { - All dogs received plated Securos Surgical TTA XGEN system. } \\
\text { - All dogs received autologous cancellous bone graft into } \\
\text { osteotomy space. } \\
\text { - All dogs received exercise restriction for } 6 \text { weeks. }\end{array}$ \\
\hline Study design: & Prospective case series \\
\hline Outcome studied: & $\begin{array}{l}\text { Complications, lameness, thigh circumference, range of motion, } \\
\text { radiographic osteoarthritis at } 6 \text { weeks, } 6 \text { months, and } 1 \text { year. }\end{array}$ \\
\hline $\begin{array}{l}\text { Main findings: } \\
\text { (relevant to PICO question): }\end{array}$ & $\begin{array}{l}\text { Postoperative complications } \mathbf{8 / 2 4} \text { (33.3\%) } \\
\text { - SSI } 2 / 24 \text { - resolved with antibiotics. } \\
\text { - } \text { Fracture of tibial tuberosity } 1 / 24 \text {. } \\
\text { - Recurrent lameness } 6 / 24 \text { dogs - responded to } \\
\text { conservative management. } \\
\text { PICO - } 0 \text { dogs had tibial diaphyseal fracture. }\end{array}$ \\
\hline Limitations: & $\begin{array}{l}\text { - Very small sample size. } \\
\text { - Utilised thigh circumference (TC) and range of motion } \\
\text { (ROM) as a measure of limb use, as opposed to peak }\end{array}$ \\
\hline
\end{tabular}




\begin{tabular}{|c|c|c|}
\hline & & $\begin{array}{l}\text { vertical forces. } \\
\text { Postoperative rehabilitation was performed on some } \\
\text { dogs and not others, which may influence TC and ROM. } \\
\text { - Clinical outcomes were subjectively measured using } \\
\text { lameness score. } \\
\text { - Follow up radiographic signs of osteoarthritis were } \\
\text { measured subjectively by one certified radiologist. }\end{array}$ \\
\hline
\end{tabular}

\begin{tabular}{|c|c|}
\hline \multicolumn{2}{|l|}{ Hans et al., (2017) tTTA } \\
\hline Population: & Large breed dog $>50 \mathrm{~kg}$ \\
\hline Sample size: & 145 stifle joints \\
\hline Intervention details: & $\begin{array}{l}91 \text { stifle joints received Securos Surgical TTA XGEN plated system } \\
54 \text { stifle joints received Tibial Plateau Leveling Osteotomy (TPLO) }\end{array}$ \\
\hline Study design: & Retrospective case-control \\
\hline Outcome studied: & $\begin{array}{l}\text { Major and minor complication rates of TTA compared to TPLO in } \\
\text { large breed dogs. The null hypothesis is that there is a difference in } \\
\text { major complications between the two techniques, based on medical } \\
\text { records. }\end{array}$ \\
\hline $\begin{array}{l}\text { Main findings: } \\
\text { (relevant to PICO question): }\end{array}$ & 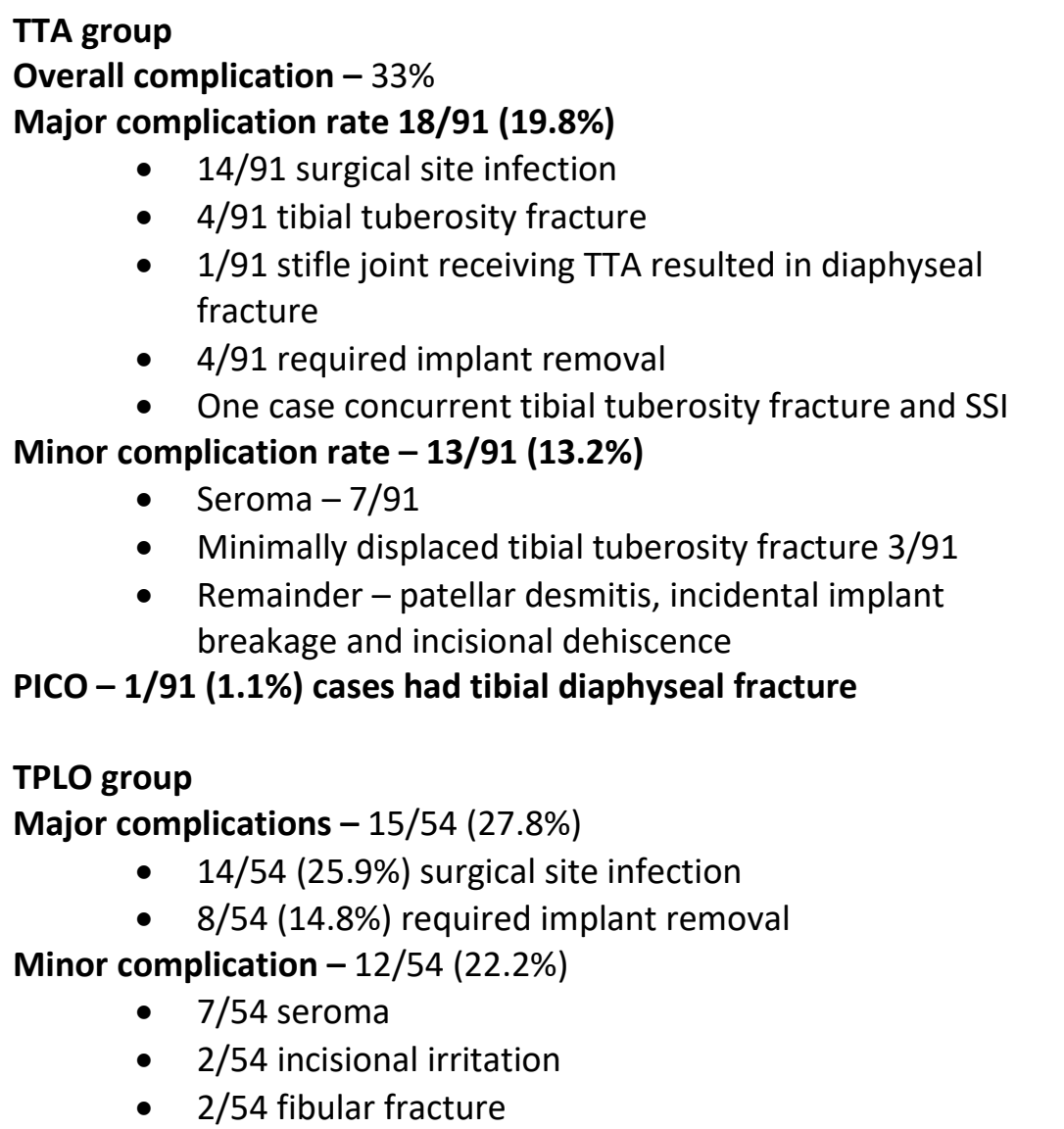 \\
\hline Limitations: & $\begin{array}{l}\text { - Surgeries were performed either by a specialist or resident. } \\
\text { However, a comparison between the two groups was not } \\
\text { analysed. }\end{array}$ \\
\hline
\end{tabular}




\begin{tabular}{|l|l|}
\hline - The power of the study was not indicated. \\
- Authors did not declare how many dogs were censored and \\
- excluded for not meeting the inclusion criteria. \\
- Leck of randomisation. \\
- Reliance on medical record accuracy. \\
receives royalties for Securos Surgical XGEN TTA system. \\
\hline
\end{tabular}

\begin{tabular}{|c|c|}
\hline \multicolumn{2}{|l|}{ Butterworth \& Kydd (2017) TTAR } \\
\hline Population: & Dogs with cruciate disease \\
\hline Sample size: & 141 clinically lame dogs, 152 stifle joints \\
\hline Intervention details: & $\begin{array}{l}\text { - All dogs received TTAR surgery. } \\
\text { - No dogs received meniscal release. } \\
\text { - All but four dogs received nanocrystalline hydroxyapatite } \\
\text { paste on osteotomy gap. }\end{array}$ \\
\hline Study design: & Case series \\
\hline Outcome studied: & $\begin{array}{l}\text { Subjective evidence of lameness and complications by clinical } \\
\text { assessment at } 3 \text { months, and validated questionnaire at }>6 \text { months } \\
\text { postoperatively. }\end{array}$ \\
\hline $\begin{array}{l}\text { Main findings: } \\
\text { (relevant to PICO question): }\end{array}$ & $\begin{array}{l}\text { - Outcome was considered clinically satisfactory in } 99 \% \text { of } \\
135 \text { dogs } 3 \text { months postoperatively and } 86 \% \text { of } 108 \text { dogs } \\
\text { in owner questionnaire. } \\
\text { - } 44 / 152(29 \%) \text { of stifle joints had medial meniscal injury } \\
\text { requiring meniscectomy } \\
\text { - } 9 / 108(8 \%) \text { developed late meniscal injury } \\
\text { - } 3 / 152(1.97 \%) \text { stifle joints developed SSI treated } \\
\text { - } 2 \text { dequately with antibiotics } \\
\text { - } 2 / 152(1.3 \%) \text { developed fracture of the tibial tuberosity } \\
\text { Tibial osteotomy gap healed at a mean and median of } \\
\text { PICO - } 1 / 152 \text { (0.66\%) developed tibial diaphyseal fracture treated by } \\
\text { internal fixation. }\end{array}$ \\
\hline Limitations: & $\begin{array}{l}\text { - Medium term follow-up was conducted using owner- } \\
\text { assessed questionnaire. } \\
\text { - Outcome was based on subjective clinical exam. } \\
\text { - } \quad \text { bumber of cage size and number of breeds were reported } \\
\text { - No control group for comparison. }\end{array}$ \\
\hline
\end{tabular}

Samoy et al., (2015) TTAR

Population: Dogs with unilateral cruciate disease 


\begin{tabular}{|c|c|}
\hline Sample size: & 50 dogs \\
\hline Intervention details: & $\begin{array}{l}\text { - All dogs received amoxicillin clavulanic acid } 8.75 \mathrm{mg} \\
\text { subcutaneously preoperatively. } \\
\text { - All dogs received TTAR surgery. } \\
\text { - All dogs received meniscal release. } \\
\text { - All dogs received hydroxylapatite bone paste onto } \\
\text { osteotomy gap. } \\
\text { - All dogs had external coaptation for } 2-3 \text { days } \\
\text { postoperatively. }\end{array}$ \\
\hline Study design: & Prospective case series \\
\hline Outcome studied: & Outcomes, minor and major complication rates. \\
\hline $\begin{array}{l}\text { Main findings: } \\
\text { (relevant to PICO question): }\end{array}$ & $\begin{array}{l}\text { - } 42 \% \text { of dogs had medial meniscal injury. } \\
\text { - } \quad \text { Subjective assessment at } 3 \text { months postoperatively showed } \\
28 \text { dogs ( } 56 \% \text { ) had an excellent outcome, } 20 \text { dogs ( } 40 \%) \text { had } \\
\text { a good outcome and two dogs ( } 4 \% \text { ) had a moderate } \\
\text { outcome (one clinical healing and one complete healing of } \\
\text { the osteotomy). } \\
\text { - } 15 / 50(30 \%) \text { of dogs had undefined minor complication } \\
\text { (undefined). } \\
\text { - } 2 / 50 \text { ( } 4 \% \text { ) dogs developed tibial tuberosity fracture; only } \\
\text { one required surgical revision. } \\
\text { - PICO - } 0 \% \text { of dogs developed tibial diaphyseal fracture }\end{array}$ \\
\hline Limitations: & $\begin{array}{l}\text { - } \quad \text { Postliminary meniscal injury not reported. } \\
\text { - Unclear if SSI did not develop or was unreported. } \\
\text { - Small sample size. } \\
\text { - Short follow-up times ( } 3 \text { months). } \\
\text { - Not tested against a control group. } \\
\text { - } \quad \text { Outcome was based on subjective clinical exam only. } \\
\text { - Conflict of interest that the developer of the technique is } \\
\text { also the primary author. }\end{array}$ \\
\hline
\end{tabular}

\section{Dyall \& Schmokel (2017) TTAR}

\begin{tabular}{|r|c|}
\hline Population: & Small breed dog - mean weight $9 \mathrm{~kg}(4.8-15 \mathrm{~kg})$ \\
\hline Sample size: & 48 stifle joints \\
\hline Intervention details: & $\begin{array}{r}\text { - } 40 \text { small breed dogs (48 stifle joints) received TTAR surgery. } \\
\\
\end{array}$ \\
& $19 / 48$ stifle joints received TTAR with Maquet hole, 29/48 \\
& had no Maquet hole. \\
& - No dogs received meniscal release. \\
& - All dogs were on exercise restriction for 6 weeks. \\
\hline
\end{tabular}




\begin{tabular}{|c|c|}
\hline Study design: & Retrospective case series \\
\hline Outcome studied: & $\begin{array}{l}\text { Lameness and postoperative complications at } 6 \text { weeks were } \\
\text { measured by clinical examination and owner questionnaire } \\
\text { assessment at mid- to long-term follow up (median } 72 \text { weeks). }\end{array}$ \\
\hline $\begin{array}{l}\text { Main findings: } \\
\text { (relevant to PICO question): }\end{array}$ & $\begin{array}{l}\text { - Major complication rate } 7 / 48(14.6 \%) \\
\text { - } \text { Infection }-1 / 48(0.02 \%) \\
\text { - } \text { Medial meniscal injury noted in } 19 / 48(40 \%) \\
\text { - } 2 / 48 \text { developed tibial tuberosity fracture requiring surgical } \\
\text { - } \quad 1 / 19(5.26 \%) \text { stifle joints with Maquet hole resulted in tibial } \\
\text { - } \quad 1 / 29(3.45 \%) \text { stifle joints without Maquet hole resulted in } \\
\text { tibial diaphyseal fracture } \\
\text { - } 2 / 48(4.17 \%) \text { stifle joints in total resulted in tibial diaphyseal } \\
\text { fracture } \\
\text { PICO- } 2 / 48(4.17 \%) \text { developed tibial diaphyseal fracture }\end{array}$ \\
\hline Limitations: & $\begin{array}{l}\text { - Small sample size. } \\
\text { - Outcome was measured by subjective client questionnaire } \\
\text { that has not been validated. } \\
\text { - } \quad \text { technique. } \\
\text { - Lack of objective outcome criteria. }\end{array}$ \\
\hline
\end{tabular}

\begin{tabular}{|c|c|}
\hline \multicolumn{2}{|l|}{ Arican et al., (2017) TTAR } \\
\hline Population: & Dogs receiving TTAR \\
\hline Sample size: & 17 \\
\hline Intervention details: & $\begin{array}{l}\text { - } 17 \text { stifle joints received TTAR surgery with Maquet hole } \\
\text { - All dogs received perioperative antibiotics amoxicillin } \\
\text { clavulanic acid ( } 8.75 \mathrm{mg} / \mathrm{kg} \text { subcutaneously) } \\
\text { - All dogs received carprofen } 4 \mathrm{mg} / \mathrm{kg} \text { perioperative analgesia } \\
\text { - } \quad \text { All dogs received } 3 \text { days postoperative meloxicam } 1 \mathrm{mg} / \mathrm{kg} \\
\text { (Intramuscular) and } 5 \text { days amoxicillin clavulanic acid } \\
\text { (undeclared dose). }\end{array}$ \\
\hline Study design: & Case series \\
\hline Outcome studied: & $\begin{array}{l}\text { Minor complications and major complications. Lameness and pain } \\
\text { assessment } 1,2 \text { and } 3 \text { months postoperatively. }\end{array}$ \\
\hline $\begin{array}{l}\text { Main findings: } \\
\text { (relevant to PICO question): }\end{array}$ & $\begin{array}{l}\text { - Minor complication rate was } 25 \% \text {. } \\
\text { - } \text { Tibial crest fracture occurred in } 2 / 17 \text { fractures; only one } \\
\text { required surgery. } \\
\text { PICO - } 0 / 17 \text { stifle joints receiving TTAR resulted in tibial diaphyseal }\end{array}$ \\
\hline
\end{tabular}




\begin{tabular}{|c|c|}
\hline & fracture. \\
\hline Limitations: & $\begin{array}{l}\text { - Meniscal findings and injury rate not reported. } \\
\text { - Minor complications were not defined. } \\
\text { - The outcomes were measured subjectively using visual } \\
\text { blinding and control but the control was not defined. } \\
\text { - Competency of the surgeon was not defined (specialist vs. } \\
\text { resident vs. general practitioner). } \\
\text { - This study has a very small sample size for assessment of } \\
\text { complication rates. } \\
\text { There is no control group to assess if there would be } \\
\text { improvement with no treatment. }\end{array}$ \\
\hline
\end{tabular}

\begin{tabular}{|c|c|}
\hline \multicolumn{2}{|l|}{ de Sousa et al., (2017) Plateless TTA } \\
\hline Population: & $\begin{array}{l}\text { Dogs that received plateless TTA with complications of tibial } \\
\text { diaphyseal fracture }\end{array}$ \\
\hline Sample size: & $\begin{array}{l}17 \text { dogs; } 11 / 17 \text { received Orthofoam-MMP procedure, } 6 / 17 \text { received } \\
\text { TTAR procedure }\end{array}$ \\
\hline Intervention details: & $\begin{array}{l}\text { All } 17 \text { dogs received revision surgery to correct tibial tuberosity } \\
\text { fracture by various forms of internal fixation. }\end{array}$ \\
\hline Study design: & Case series \\
\hline Outcome studied: & $\begin{array}{l}\text { Outcome, major and minor complication following repair of tibial } \\
\text { diaphyseal fracture secondary to complication of plateless TTA } \\
\text { techniques. }\end{array}$ \\
\hline $\begin{array}{l}\text { Main findings: } \\
\text { (relevant to PICO question): }\end{array}$ & $\begin{aligned} \text { - } & \text { Overall complication rate }-8 / 17(47 \%) \\
\text { - } & \text { Minor complication rate }-3 / 17(17.6 \%) \\
\text { - } & \text { Major complication rate }-5 / 17(29.4 \%) \\
\text { - } & \text { Surgical site infection }-4 / 17(23.5 \%) \\
\text { PICO } & \\
\text { - } & \text { TTAR } \\
\text { - } & N=6 \\
\text { - } & \text { Major complication }-0 / 6 \\
\text { - } & \text { Minor complication }-2 / 6\end{aligned}$ \\
\hline Limitations: & $\begin{array}{l}\text { - Small sample size }-6 \text { TTAR and } 11 \text { Orthofoam-Modified } \\
\text { Maquet Procedure. } \\
\text { Variation in surgeons managing complication - six } \\
\text { boarded specialist vs. four RCVS Certificate qualified } \\
\text { veterinary surgeons. } \\
\text { - Owner compliance to original procedure was not } \\
\text { measured or accounted for. } \\
\text { - Incidence of these tibial diaphyseal fractures could not } \\
\text { be determined. } \\
\text { - No comparison with fracture complications from } \\
\text { traditional TTA methods. }\end{array}$ \\
\hline
\end{tabular}




\section{Appraisal, application and reflection}

Since the advent of tTTA in 2002 (Montavon et al., 2002), various second generation TTA procedures have been invented. These techniques (TTAR, Orthofoam-MMP, MMT, TTA-2) utilise custom saw guides to aid the surgeon in producing an incomplete osteotomy of the tibial tuberosity. They have different implant designs, and they eliminate the use of a supporting plate (Ness 2016 Samoy et al., 2015, Bleakley 2015, Brunel et al., 2013). These techniques are generally aimed at the non-specialist surgeon and are marketed to be simpler than tTTA while offering comparable results. A recent case series of 17 dogs (de Sousa et al, 2017) suggests that tibial diaphyseal fractures may be a well-recognised catastrophic complication of second generation TTA techniques. However, the study design could not evaluate the actual incidence or prevalence of this complication.

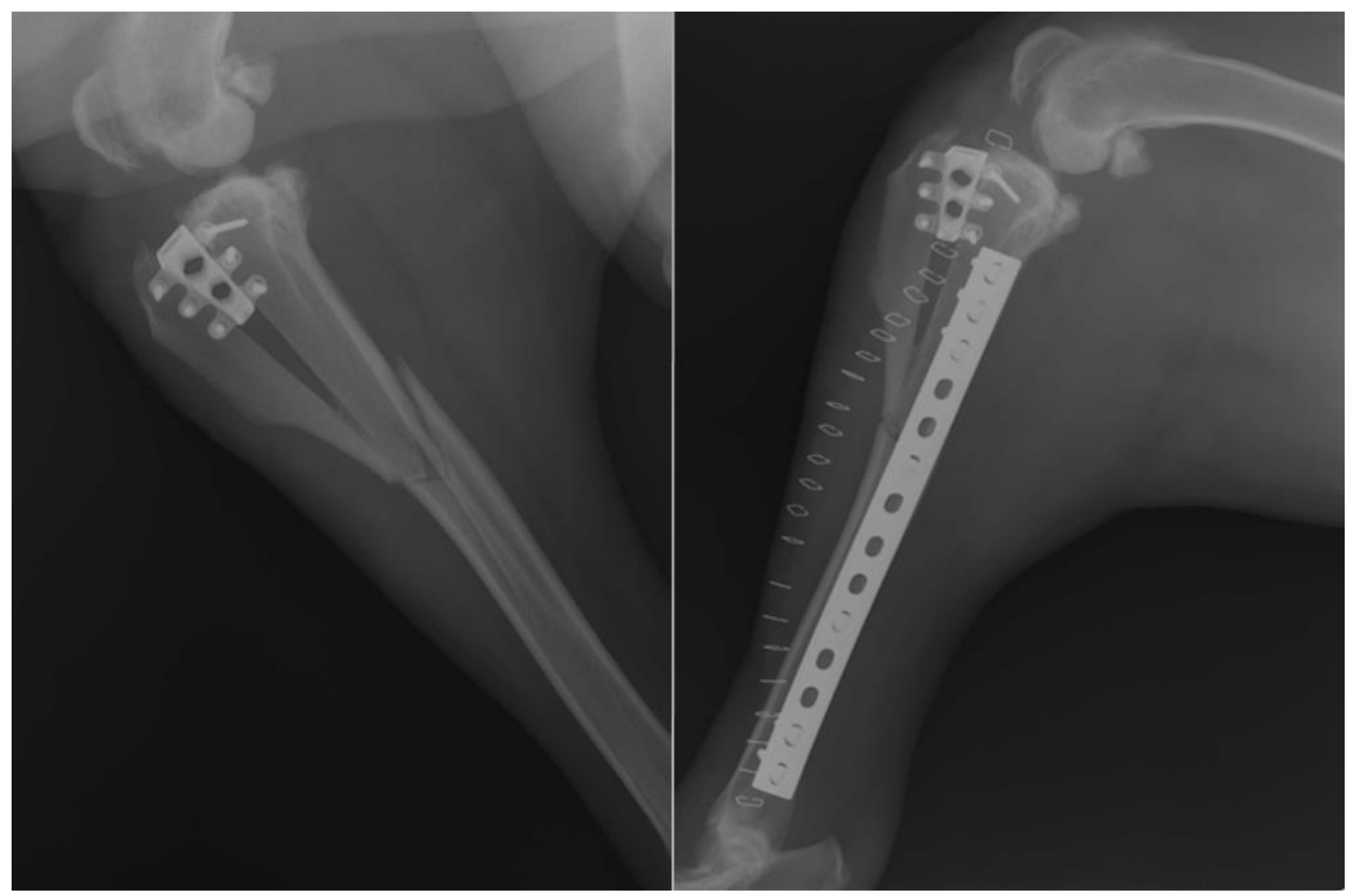

Figure 1: Example of tibial diaphyseal fracture as a complication of TTAR technique. 


\begin{tabular}{|c|c|c|c|c|}
\hline TTA type & Study & $\begin{array}{l}\text { Tibial Diaphyseal } \\
\text { fractures }\end{array}$ & $\begin{array}{l}\text { Tibial Tuberosity } \\
\text { Fracture }\end{array}$ & EBVM level \\
\hline tTTA & Costa et al., (2017) & $3 / 1613(0.18 \%)$ & $8 / 1613(0.5 \%)$ & Level 4 \\
\hline tTTA & $\begin{array}{l}\text { Danielson et al., } \\
\text { (2016) }\end{array}$ & $1 / 148(0.7 \%)$ & $4 / 148(2.7 \%)$ & Level 4 \\
\hline tTTA & Proot \& Corr (2013) & $0 / 167(0 \%)$ & $3 / 167(1.8 \%)$ & Level 4 \\
\hline tTTA & $\begin{array}{l}\text { de Lima Dantas et al., } \\
\text { (2016) }\end{array}$ & $0 / 307(0 \%)$ & $\begin{array}{l}2 / 36(5.56 \%) \text { in } \\
\text { Boxers } \\
1 / 271(0.4 \%) \text { in } \\
\text { non-Boxers } \\
3 / 307(1 \%) \text { in } \\
\text { total }\end{array}$ & Level 3 \\
\hline tTTA & Kiefer et al., (2015) & $\begin{array}{l}2 / 88(2.27 \%) \text { in } \\
\text { bilateral TTA } \\
\text { group. } \\
\text { 0/88 in unilateral } \\
\text { TTA group }\end{array}$ & $\begin{array}{l}\text { 9/99 }(9.1 \%) \text { in } \\
\text { bilateral TTA } \\
\text { group } \\
1 / 88(1.13 \%) \text { in } \\
\text { unilateral TTA } \\
\text { group }\end{array}$ & Level 3 \\
\hline tTTA & $\begin{array}{l}\text { McDonald et al., } \\
\text { (2013) }\end{array}$ & $0 / 24(0 \%)$ & $1 / 24(5.2 \%)$ & Level 4 \\
\hline tTTA & Hans et al., (2017) & $1 / 91 \%(1.1 \%)$ & $4 / 91(4.4 \%)$ & Level 3 \\
\hline TTAR & $\begin{array}{l}\text { Butterworth } \quad \& \\
\text { Kydd., (2017) }\end{array}$ & $1 / 152(0.7 \%)$ & $2 / 152(1.3 \%)$ & Level 4 \\
\hline TTAR & Samoy et al., (2015) & $0 / 50(0 \%)$ & $2 / 50(4 \%)$ & Level 4 \\
\hline TTAR & $\begin{array}{l}\text { Dyall \& Schmokel } \\
\text { (2017) }\end{array}$ & $2 / 48(4.17 \%)$ & $2 / 48(4.17 \%)$ & Level 4 \\
\hline TTAR & Arican et al., (2017) & $0 / 17(0 \%)$ & $2 / 17(11.7 \%)$ & Level 4 \\
\hline
\end{tabular}

Table 2: Based on recent ( $<5$ years) studies relevant to the PICO, tibial diaphyseal fractures are reported in the table. While not directly related to the PICO, tibial tuberosity fractures are also included.

From this table, the rate of tibial diaphyseal fractures from tTTA $(0-2.3 \%)$ compared to TTAR $(0-4.17 \%)$ are roughly comparable. In the TTAR group, Dyall \& Schmokel's (2017) report of $4.17 \%$ tibial diaphyseal fractures using TTAR on small breed dogs $(<15 \mathrm{~kg})$ was notably higher than the other three studies. The higher fracture rate was attributed to the relatively large cage size, which may have overwhelmed the elasticity of the cranial tibial cortex, predisposing to fracture. If small breed dogs were excluded from the comparison then the rate of tibial diaphyseal fractures for TTAR would be $0-0.7 \%$, which is within the published limits for tTTA (02.3\%). A two-tailed T test (https://www.socscistatistics.com/tests/studentttest/Default2.aspx) comparing TTAR $(0,0,0.7,4.17)$ vs tTTA $(0.18,0.7,0,0,2.27,0,1.1)$ reveals $t$-value of 0.7246 and $p$-value of 0.487105 , showing a lack of significance at $\mathrm{p}<0.05$, although the sample size is very small.

Interestingly, tibial tuberosity fractures, while not directly related to the PICO, occur more commonly across studies. In tTTA, a complete osteotomy of the tuberosity is held in place by a forked or screw plate. Fractures are generally attributed to poor plate and cage positioning, as well as narrow tuberosity width postosteotomy (Costa et al., 2017). In TTAR, the osteotomy is incomplete leaving the distal hinge intact. 
Fractures through the distal hinge were considered as tibial tuberosity fractures across the TTAR studies (Butterworth \& Kydd (2017), Samoy et al., (2015), Dyall \& Schmokel (2017), Arican et al., (2017)). Despite the added risk of the distal hinge fracture for TTAR, the tibial tuberosity fracture rate of $0.5-9.1 \%$ for tTTA and $1.3-11.7 \%$ for TTAR does not differ significantly at $p<0.05$, with two-tailed T test showing $t$-value of -0.91676 and $p$-value 0.378929. In addition, the 2/50 tibial tuberosity fractures in Samoy et al. (2015) happened before the development of a dedicated saw guide. The TTAR has since been modified to exclude the use of the Maquet hole at the distal end of the incomplete osteotomy while utilising a longer osteotomy. This is facilitated by a dedicated saw guide, allowing an accurate cut. It is yet unknown if this will lead to reduced tuberosity and diaphyseal fractures.

Proot \& Corr's (2013) clinical audit suggests that it takes 22 tTTA procedures to gain acceptable competence. To date, a similar clinical audit has not been published for TTAR and it is unknown if major complications such as tibial fractures are more common in the hands of inexperienced veterinary surgeons. It is also unclear from the data how often tibial fractures are due to faults in the technique or faults in the postoperative care.

It should be noted that all evidence that met the inclusion criteria for this PICO is of low grade evidence and to date no head-to-head control trials have been done comparing tTTA to TTAR or other second-generation plateless techniques. Future studies could focus on which perioperative factors might have an impact on fracture complications. For example, the experience of the veterinary surgeon, the use of bone grafting, which may accelerate healing, the size and age of the patient, which may influence bone elasticity, as well as owner compliance and protective effect of external coaptation in the early postoperative period. Based on current evidence however, the rate of tibial fractures does not differ between tTTA and TTAR. Until data to the contrary becomes available, veterinary surgeons performing either technique should be cognizant of their ability to perform complex osteotomy, as well as audit their complications and compare them against published limits.

\section{Methodology Section}

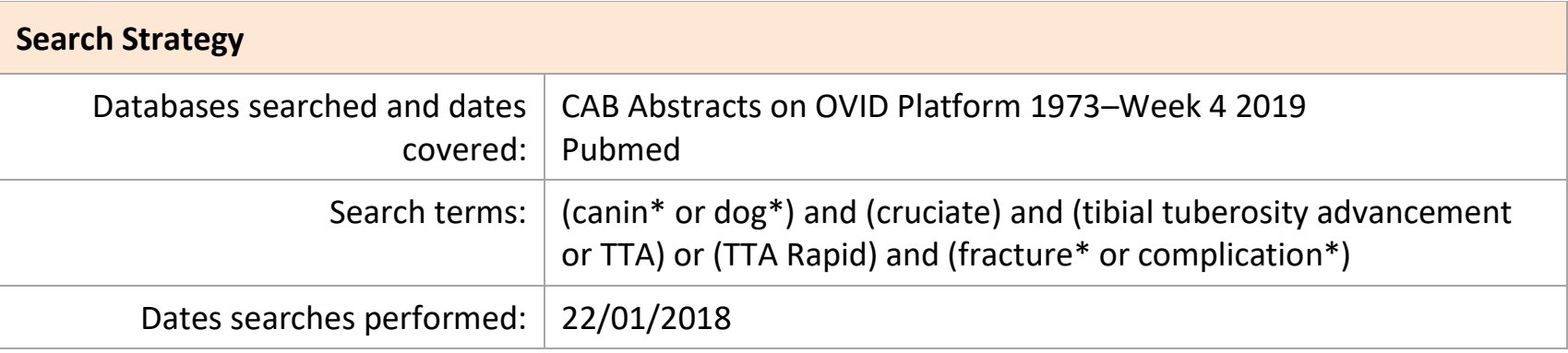

\section{Exclusion / Inclusion Criteria}

Exclusion: $C A B$ Abstracts via the Ovid platform covering from 1973 to 2013 weeks 1.

Pubmed with publication dates $>5$ years

Non-english articles

Articles with contents irrelevant to PICO.

Inclusion: All TTAR papers and tTTA related papers with data pertaining to tibial fractures and complications. 


\begin{tabular}{|l|c|c|c|c|c|}
\hline \multicolumn{2}{|l|}{ Search Outcome } & \multicolumn{2}{|l|}{} \\
\hline Database & $\begin{array}{c}\text { Number of } \\
\text { results }\end{array}$ & $\begin{array}{c}\text { Excluded - } \\
\text { non English }\end{array}$ & $\begin{array}{c}\text { Excluded - } \\
\text { publications > 5 } \\
\text { years old }\end{array}$ & $\begin{array}{c}\text { Excluded - } \\
\text { irrelevant to } \\
\text { PICO }\end{array}$ & $\begin{array}{c}\text { Total relevant } \\
\text { papers }\end{array}$ \\
\hline CAB Abstracts & 48 & 11 & 15 & 10 & 12 \\
\hline Pubmed & 37 & 0 & 17 & 11 & 9 \\
\hline Total relevant papers when duplicates removed & & & 12 \\
\hline
\end{tabular}

\section{CONFLICT OF INTEREST}

The author declares no conflicts of interest.

\section{REFERENCES}

1. Arican, M., Parlak, K., \& Şahin, H. (2017). Evaluation and application of the TTA-rapid method in dogs with cranial cruciate ligament rupture. Acta Veterinaria, 67(2), 238-253. http://doi.org/10.1515/acve$\underline{2017-0020}$

2. Brunel, L., Etchepareborde, S., Barthélémy, N., et al. (2013) Mechanical testing of a new osteotomy design for tibial tuberosity advancement using the modified Maquet technique. Veterinary and Comparative Orthopaedics and Traumatology 26, 47-53.

3. Bleakley, S. (2015) TTA-2: Research Prokects, Kyon Symposium

4. Butterworth, S. J., \& Kydd, D. M. (2017). TTA-Rapid in the treatment of the canine cruciate deficient stifle: short- and medium-term outcome. Journal of Small Animal Practice, 58(1), 35-

41. http://doi.org/10.1111/jsap.12610

5. Costa, M., Craig, D., Cambridge, T., Sebestyen, P., Su, Y., \& Fahie, M. A. (2017). Major complications of tibial tuberosity advancement in 1613 dogs. Veterinary Surgery, 46(4), 494500. http://doi.org/10.1111/vsu.12649

6. Danielson, B., Barnhart, M., Watson, A., Kennedy, S., \& Naber, S. (2016). Short-Term Radiographic Complications and Healing Assessment of Single-Session Bilateral Tibial Tuberosity Advancements. Journal of the American Animal Hospital Association, 52(2), 109114. http://doi.org/10.5326/JAAHA-MS-6194

7. de Sousa, R., Egan, P., Parsons, K., Butterworth, S., Calvo, I., Roch, S., \& Moores, A. P. (2017). Treatment of tibial diaphyseal fractures following plateless tibial tuberosity advancement to manage 
cranial cruciate disease. Journal of Small Animal Practice, 58(7), 372-

379. http://doi.org/10.1111/jsap.12673

8. de Lima Dantas, B., Sul, R., Parkin, T., \& Calvo, I. (2016). Incidence of complications associated with tibial tuberosity advancement in boxer dogs. Veterinary and Comparative Orthopaedics and Traumatology, 29(1), 39-45. http://doi.org/10.3415/VCOT-15-02-0036

9. Dyall, B., \& Schmökel, H. (2017). Tibial tuberosity advancement in small-breed dogs using TTA Rapid implants: complications and outcome. Journal of Small Animal Practice, 58(6), 314322. http://doi.org/10.1111/jsap.12654

10. Hans, E. C., Barnhart, M. D., Kennedy, S. C., \& Naber, S. J. (2017). Comparison of complications following tibial tuberosity advancement and tibial plateau levelling osteotomy in very large and giant dogs $50 \mathrm{~kg}$ or more in body weight. Veterinary and Comparative Orthopaedics and Traumatology, 30 (4), 299-305. http://doi.org/10.3415/VCOT-16-07-0106

11. Kiefer, J. E., Langenbach, A., Boim, J., Gordon, S., \& Marcellin-Little, D. J. (2015). Single-stage bilateral tibial tuberosity advancement for treatment of bilateral canine cranial cruciate ligament deficiency. Veterinary and Comparative Orthopaedics and Traumatology, 28 (3), 215219. http://doi.org/10.3415/VCOT-14-08-0130

12. MacDonald, T. L., Allen, D. A., \& Monteith, G. J. (2013). Clinical assessment following tibial tuberosity advancement in 28 stifles at 6 months and 1 year after surgery. Canadian Veterinary Journal, 54 (3), 249-254.

13. Montavon PM, Damur DM, Tepic S (2002). Advancement of the tibial tuberosity for the treatment of cranial cruciate deficient canine stifle. Proceedings of the 1st World Orthopedic Veterinary Congress; 2002 September 5-8; Munich, Germany. 152.

14. Ness M (2016) The Modified Maquet Procedure (MMP) in Dogs: Technical Development and Initial Clinical Experience, Journal of the American Animal Hospital Association, 52 (4), 242-250.

15. Proot, J. L. J., \& Corr, S. A. (2013). Clinical audit for the tibial tuberosity advancement procedure. Veterinary and Comparative Orthopaedics and Traumatology, 26 (4), 280284. http://doi.org/10.3415/VCOT-12-04-0052

16. Samoy, Y., Verhoeven, G., Bosmans, T., Van der Vekens, E., de Bakker, E., Verleyen, P., \& Van Ryssen, B. (2015). TTA Rapid: Description of the Technique and Short Term Clinical Trial Results of the First 50 Cases. Veterinary Surgery, 44 (4), 474-484. http://doi.org/10.1111/j.1532-950X.2014.12298.x 


\section{EVIIDEFeE

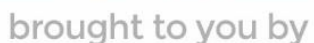 \\ RCVS KNOWLEDGE}

\section{Intellectual Property Rights}

Authors of Knowledge Summaries submitted to RCVS Knowledge for publication will retain copyright in their work, and will be required to grant RCVS Knowledge a non-exclusive license of the rights of copyright in the materials including but not limited to the right to publish, re-

publish, transmit, sell, distribute and otherwise use the materials in all languages and all media throughout the world, and to license or permit others to do so.

\section{Disclaimer}

Knowledge Summaries are a peer-reviewed article type which aims to answer a clinical question based on the best available current evidence. It does not override the responsibility

of the practitioner. Informed decisions should be made by considering such factors as individual clinical expertise and judgement along with patient's circumstances and owners' values. Knowledge Summaries are a resource to help inform and any opinions expressed within the Knowledge Summaries are the author's own and do not necessarily reflect the view of the RCVS Knowledge. Authors are responsible for the accuracy of the content. While the

Editor and Publisher believe that all content herein are in accord with current recommendations and practice at the time of publication, they accept no legal responsibility

for any errors or omissions, and make no warranty, express or implied, with respect to material contained within.

For further information please refer to our Terms of Use.

RCVS Knowledge is the independent charity associated with the Royal College of Veterinary Surgeons (RCVS). Our ambition is to become a global intermediary for evidence based veterinary knowledge by providing access to information

that is of immediate value to practicing veterinary professionals and directly contributes to evidence based clinical decision-making.

https://www.veterinaryevidence.org/

RCVS Knowledge is a registered Charity No. 230886.

Registered as a Company limited by guarantee in England and Wales No. 598443.

Registered Office: Belgravia House, 62-64 Horseferry Road, London SW1P 2AF

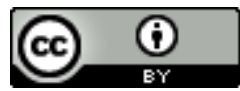

This work is licensed under a Creative Commons Attribution 4.0 International License. 